\title{
Modern Coding Sequences Are in the Periodic-to-Chaotic Transition
}

S. Ohno

\section{A. Introduction}

It seems as though biologists are extraordinarily fond of randomness. A population is defined as one, randomly mating, interbreeding unit, although truly random mating would hardly be practiceable in a reasonably large population. Similarly, spontaneous mutations are viewed as randomly sustained base substitutions, in spite of our knowledge of mutational hot spots. I suspect that this extraordinarily strong belief in randomness stems from our too strong faith in the power of natural selection. The unpredictable world of randomness is the world of chaos. Yet in recent times, there has been increasing realization that there is order in chaos as well. This realization started with three equations by Laurenz to describe meteorological phenomena. No one would dispute the unpredictability of weather. Yet, these three equations describing heat reflected by the earth and frictions caused by rotation of the earth revealed the presence of the strange attractor. The presence of the attractor, no matter how strange, is a sure indication of order. Thus, Feigenbaum's conjecture on chaos came about [1].

There are many different ways of viewing these developments. Nevertheless, I will present one version pertinent to the present discussion: the chaotic state is the degenerate form of the ordered (periodical) state, and this degeneration is due primarily to progressive step-wise increase of the original periodicity. Keeping the above in mind, now let us exam-

Beckman Research Institute of the City of Hope, Duarte, California, U.S.A. ine the 173-codon-long chicken lens $\alpha \mathrm{A}$ crystallin which is primarily made of $\beta$ sheet structures [2].

\section{B. CCTG Tetramer as the Primordial Repeating Unit of the Crystallin Coding Sequence}

As shown at the top of Fig. 1, this GC rich coding sequence contained more pyrimidines than purines because of the abundance of $\mathrm{C}(32.4 \%)$. After this realization, the frequency distribution of $\mathrm{C}$ containing dimers $(\mathrm{C} \mathrm{X}$ and $\mathrm{X} C$ ) were obtained. The procedure forced $\mathrm{C} \mathrm{C}$ dimers to be overrepresented, for the $\mathrm{C} \mathrm{C}$ $\mathrm{C}$ trimer was counted as $2 \mathrm{C} \mathrm{C}$ dimers. This was because th $\mathrm{C} \mathrm{C} \mathrm{X} \mathrm{trimer} \mathrm{C} \mathrm{C} \mathrm{A,}$ e.g., had to be counted as a $1 \mathrm{C} \mathrm{C}$ and a $1 \mathrm{C} \mathrm{A}$ dimer. If $\mathrm{C} \mathrm{C} \mathrm{C}$ was regarded as 1 $\mathrm{C} \mathrm{C}$ dimer, the recurrence rate of the $\mathrm{C} \mathrm{C}$ dimer is reduced to $47 \mathrm{X}$. Since all sequences, no matter how short, are translatable by three reading frames, $1 / 3$ of them should serve as Pro codons C C X. This predicts the presence of 16 Pro $(9 \%)$ in this protein. Indeed, there were 14 Pro residues. Next to $\mathrm{C} C$, the more frequently recurring $\mathrm{C}$-containing dimers were $\mathrm{T}$ C (41 X), C T (39 X), and C A (39 X). The above suggested relative abundance of Ser and Leu but not of Gln and His, for $1 / 3$ of $39 \mathrm{C} \mathrm{A} \mathrm{X}$ are to be split evenly between Gln codons $C A$ G, C A A, and the His codons $\mathrm{C} \mathrm{A} \mathrm{C}$ and $\mathrm{C} \mathrm{A} \mathrm{T.} \mathrm{Indeed,}$ there were $5 \mathrm{Gln}$ and $6 \mathrm{His}$ residues. The very fact that the amino acid composition of the protein is fairly predictable by recurrent rates of base dimers in its coding sequence immediately places in grave doubt the conventional wisdom of genes 
173-CODON-LONG

$$
\text { A } T / G C=0.78
$$

$$
A: 109, G: 123, T: 119, \quad C: 168(32.4 \%)
$$

\begin{tabular}{|c|c|c|c|c|c|c|c|c|c|}
\hline & & & SIRJBUII & $\mathrm{ON}-\mathrm{OE} \mathrm{C}$ & -DI & & & & \\
\hline$C$ C: $\quad 51$ & & CI: $\quad 39 X$ & & $: 39 x$ & & C G: & $23 x$ & & \\
\hline 14 PR0 & & 15 LEU & & $1 N \& 6$ & & $13 \mathrm{ARC}$ & & & \\
\hline & & & SIRIBUIU & ONLOE X & -DI & & & & \\
\hline C C: 51 & & ${ }_{25 \text { SER }} 41 X$ & $\begin{array}{ll}A \\
6\end{array}$ & : $27 x$ & & $\frac{G C:}{4 \text { ALA }}$ & $28 \times$ & & \\
\hline & & & AGE OE S & YNONYMO & 60 & & & & \\
\hline $\begin{array}{r}17 \\
4 .\end{array}$ & $\begin{array}{l}+8 \text { SER } \\
\text { I C X }\end{array}$ & & & $\begin{array}{l}3 \text { LEU } \\
\text { C T X }\end{array}$ & & & & $\begin{array}{l}3 \text { ARE } \\
C G X\end{array}$ & \\
\hline TRIMER & & ODON & TRIMER & & DON & & TRIMER & & CODOI \\
\hline 18 & $T \subset C$ & 10 & 15 & C T G & 9 & & 9 & $C G G$ & 4 \\
\hline 14 & $T \subset T$ & 4 & 12 & C T C & 3 & & 7 & $C G C$ & 4 \\
\hline 5 & $T C A$ & 1 & 8 & $C T T$ & 0 & & 5 & $C G T$ & 2 \\
\hline 4 & $T \subset G$ & 2 & 4 & $C T A$ & 0 & & 2 & $C G A$ & 0 \\
\hline & $A G X$ & & & TIX & & & & $A G X$ & \\
\hline 10 & $A G C$ & 7 & 8 & T T G & 3 & & 8 & $A G G$ & 2 \\
\hline 6 & $A G T$ & 1 & 4 & T T A & 0 & & 5 & $A G A$ & 1 \\
\hline
\end{tabular}

Fig. 1. At the top, the AT/GC ratio and base composition of the 519-base-long chicken $\alpha \mathrm{A}$-crystallin coding sequence [2] are given, followed by the recurrent rates of $C X$ and $X \mathrm{C}$ dimers. The rate for the $\mathrm{C} \mathrm{C}$ dimer is an overestimate for the reason given in the text. In the case of Leu, Gln, His, and $\mathrm{Thr}$, the recurrence of a relevant dimer divided by 3 gives a resonable estimation of the number of amino acids. At the bottom, 6 codons each for Ser, Leu, and Arg are shown in three vertical columns. The recurrent rates of each as a trimer and as a codon are shown. In each instance, the most preponderant among the synonymous codons also recurred most frequently as the base trimer

evolving by natural selection operating upon individual codons. Indeed, three columns at the bottom of Fig. 1 show that with regard to Ser, Leu, and Arg, encodable by 6 codons each, preponderant among the synonymous codons sharing the first two bases invariably is the one that recurred most frequently as base trimer. Thus, codon usages too are determined merely by recurrent rates of pertinent base trimers. Figure 1 and data not shown also suggested that the most frequently recurring base tetramer should be C C T G. This was due to the fact that the $21 \mathrm{X}$ recurring trimer $\mathrm{C} \mathrm{C} \mathrm{T}$ and the $15 X$ recurring $C \mathrm{~T} \mathrm{G}$ overlap with each other.
Indeed, $C \mathrm{C} \mathrm{T} \mathrm{G}$ was the most frequently recurring base tetramer; $9 \mathrm{X}$ recurrence (Fig. 2). This tetramer was translated in all three different reading frames to encode two Pro, five Leu, and one each of Trp and Cys. As might be deduced from Fig. 1, the next most frequently recurring base tetramer was $7 \mathrm{X}$ recurring $\mathrm{T} \mathrm{C} \mathrm{T} \mathrm{C}$ as shown boxed in Fig. 2. T C T C, however, can be regarded as two successive $T \mathrm{C}$ dimers. Nevertheless, this tetramer would soon be mentioned again. How significant was the 9 $\mathrm{X}$ recurrence of C C T G? The expected recurrence rate of this tetramer can be computed in two different ways. If based upon the $15 \mathrm{X}$ recurrent rate of $\mathrm{C} \mathrm{T} \mathrm{G}$, 


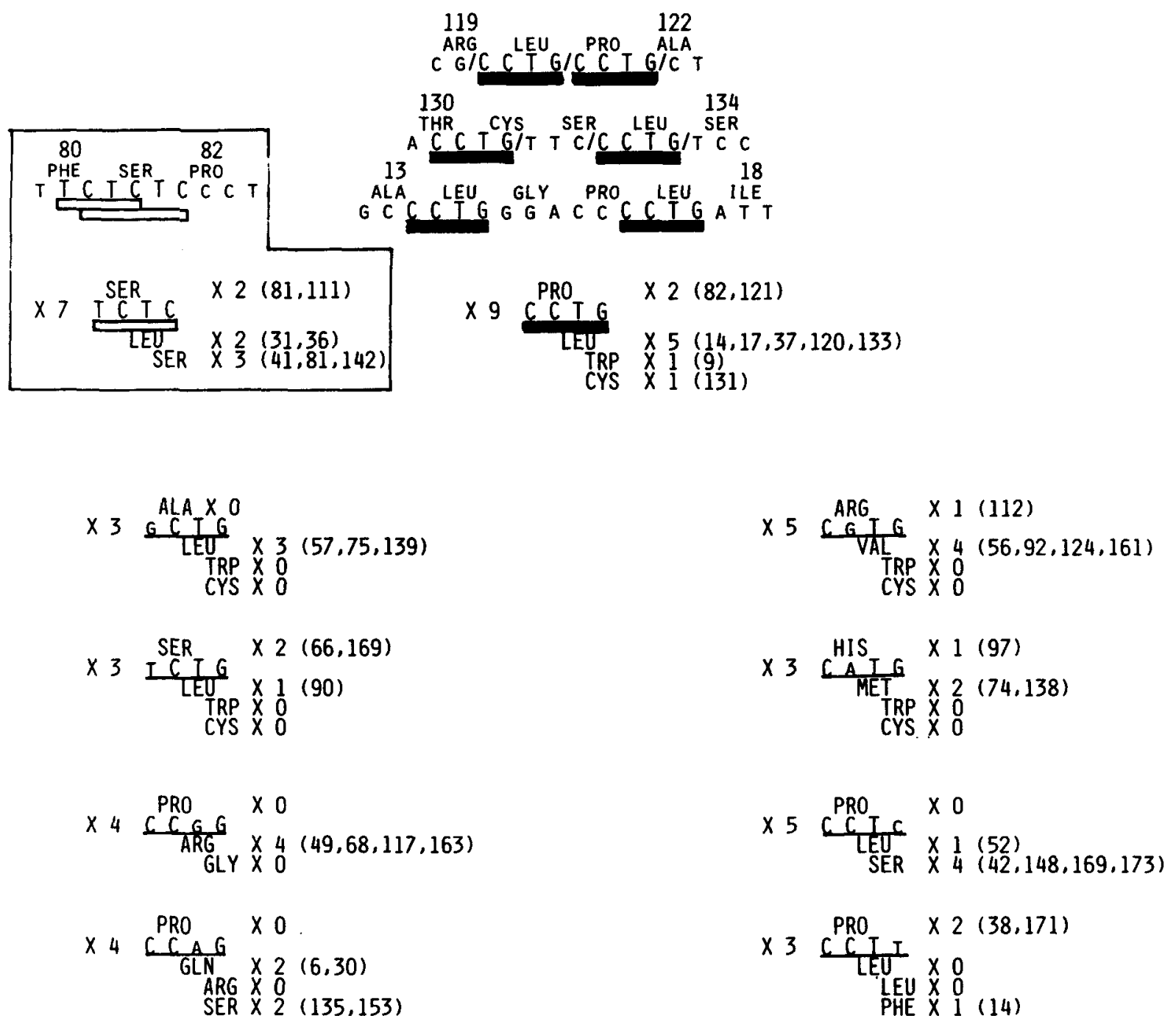

Fig. 2. C C T G as the primordial heptamer is underlined by the thick solid bar. It recurred 9 $\mathrm{X}$ and was translated in three different reading frames as shown in the upper center stage. In its 1 st reading frame, it encoded 2 Pro (the positions of these Pro in the amino acid sequence are shown in parentheses), 5 Leu in its 2nd, and 1 each of Trp and Cys in its 3rd reading frames. Shown at the top are three pairs of C C T G that recurred in succession. Placed inside the box at the left is the 2 nd most frequently recurring base tetramer T C T C that recurred $7 \mathrm{X}$. This, however, is a $\mathrm{T} \mathrm{C}$ dimer X 2 and in one place a $\mathrm{T} \mathrm{C}$ dimer recurred three times in succession. Shown in two columns near the bottom are 8 of the 12 single-base-substituted derivatives of $G$ C T G that recurred $3 \mathrm{X}$ or more

the expected recurrent rate for $\mathrm{C} \mathrm{C} \mathrm{T} \mathrm{G}$ becomes $0.324 \times 15=4.86$. As shown at the top of Fig. 1, 0.324 of the 519 bases were Cs. If based upon the $21 \mathrm{X}$ recurrence of the $\mathrm{C} \mathrm{C} \mathrm{T} \mathrm{trimer,} \mathrm{the} \mathrm{expected}$ recurrence rate of $\mathrm{C} \mathrm{C} \mathrm{T} \mathrm{G} \mathrm{now} \mathrm{becomes}$ $21 \times 0.237=4.9$. Clearly the $9 \times$ recurrence of the C C T G tetramer was not by chance. Due to single base substitutions affecting one or the other of four positions, C C T G was expected to yield 12 different kinds of single-base-substituted derivatives. As shown in the bottom half of Fig. 2, 8 of them recurred 3 to 5 times, while the remainder recurred twice each. It follows then that not counting several overlapped bases twice, C C T G and its single-base-substituted derivatives occupied $35 \%$ of the entire coding sequence.

It would thus appear that the $\alpha \mathrm{A}$-crystallin coding sequence was ultimately derived from C C T G tetrameric repeats 

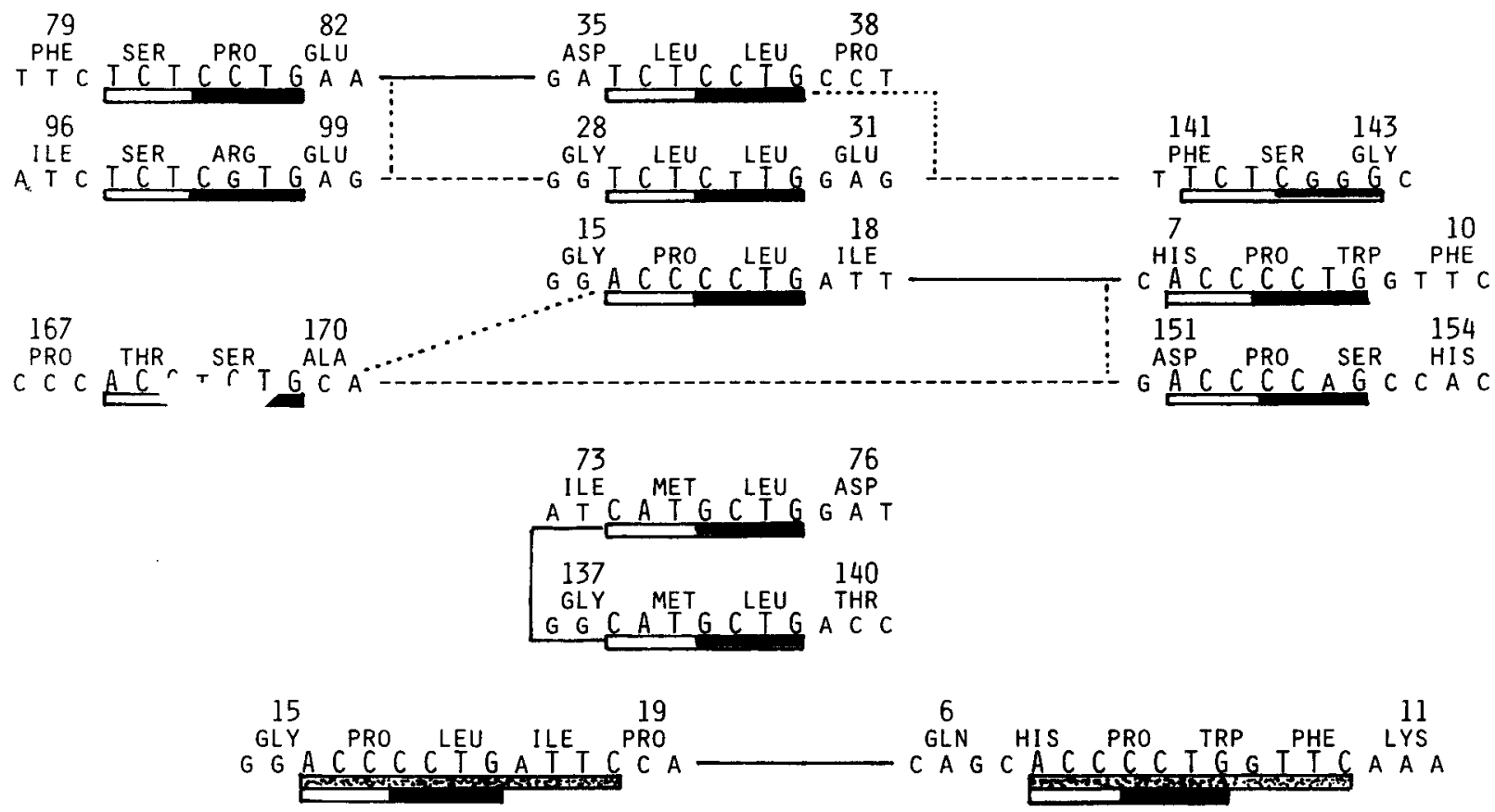

\section{THE PERIODICITY DECAY IS AGAIN BY $4,7,11$}

Fig. 3. Shown in the 1st and 3rd rows are two pairs of identical C C T G containing heptamers, while shown in the 3rd and 4th rows are each one's respective single-base-substituted copies. Identical heptamers are connected by the solid line and single-base-substituted derivatives by broken lines. Those translated in the 1st reading frame are shown in the left column, while the center column contains those translated in 2 nd reading frame, and the right column those in 3 rd reading frame. Two identical G C T G-containing heptamers, both translated in the 2 nd reading frame, are shown in the 5th and 6th rows. Two identical heptamers shown in the 3rd row were actually parts of 11-base-long repeating units as shown at the bottom

\section{MUSICAL TRANSFORMATION OF A C C C C T G HEPTAMERIC REPEATING UNIT IN CHICKEN ALPH-A-CRYSTALLIN}

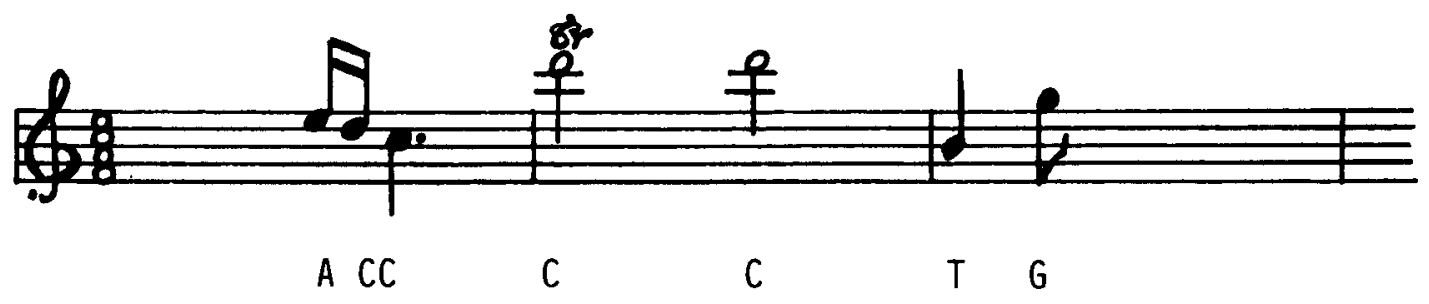

Fig. 4. The particular musical transformation given to the recurring heptamer A C C C C T G according to the set rule previously put forward [5]

that existed in the prebiotic world of eons ago [3]. Three consecutive copies of C C $T G$ should have given the tetrapeptidic periodicity Pro-Ala-Cys-Leu to the original peptide chain. Indeed, the 120 th and 121 st Leu-Pro of the chicken $\alpha$ A-crys- tallin were still encoded by two consecutive copies of $\mathrm{C} \mathrm{C} \mathrm{T} \mathrm{G} \mathrm{as} \mathrm{shown} \mathrm{at} \mathrm{the}$ very top of Fig. 2. As the periodicity decayed in the periodic-to-chaotic transition, the original exact tetrameric periodicity should have yielded to longer and 
CHICKEN LENS ALPHA-A CRYSTALLIN
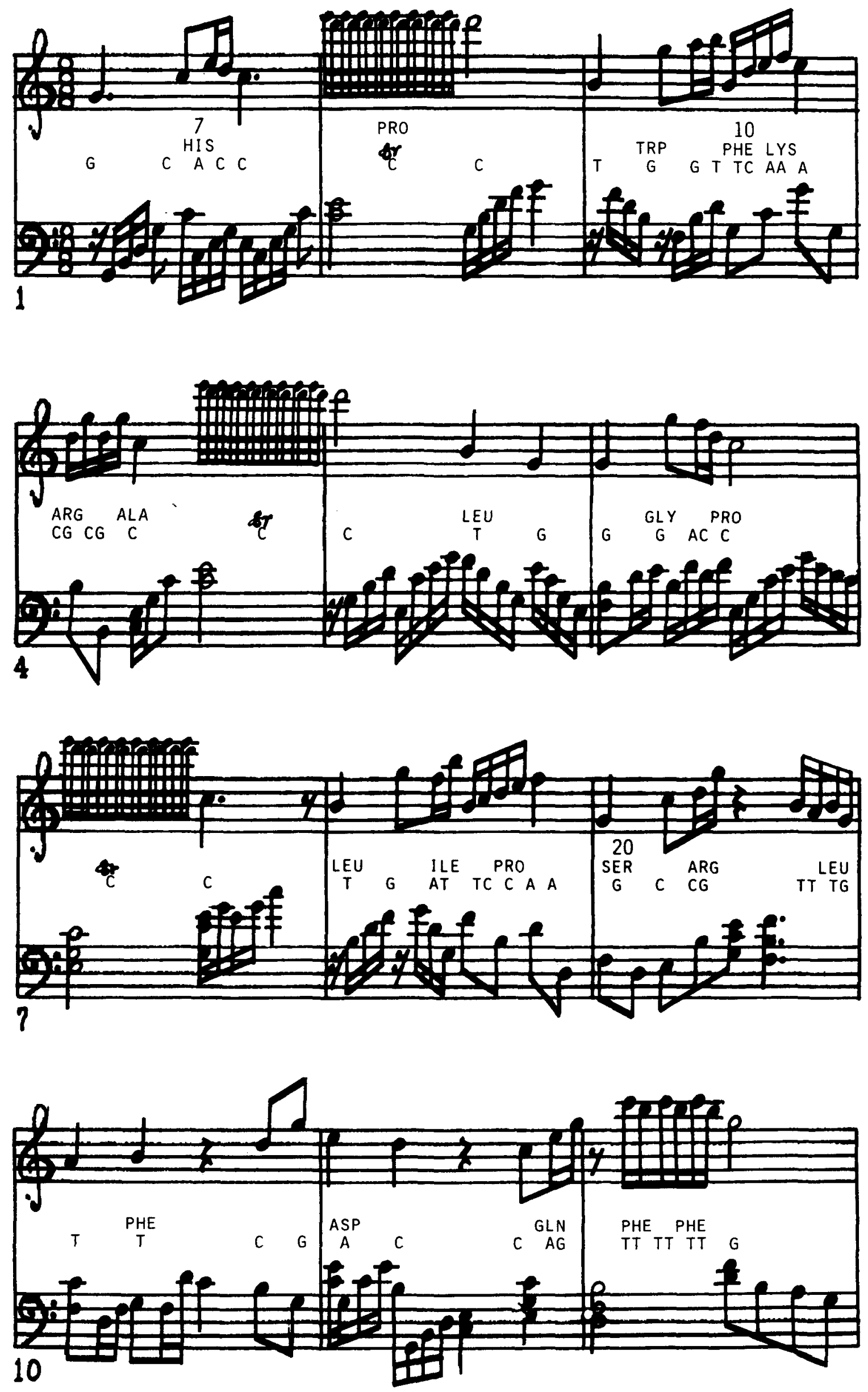

Fig. 5 a 

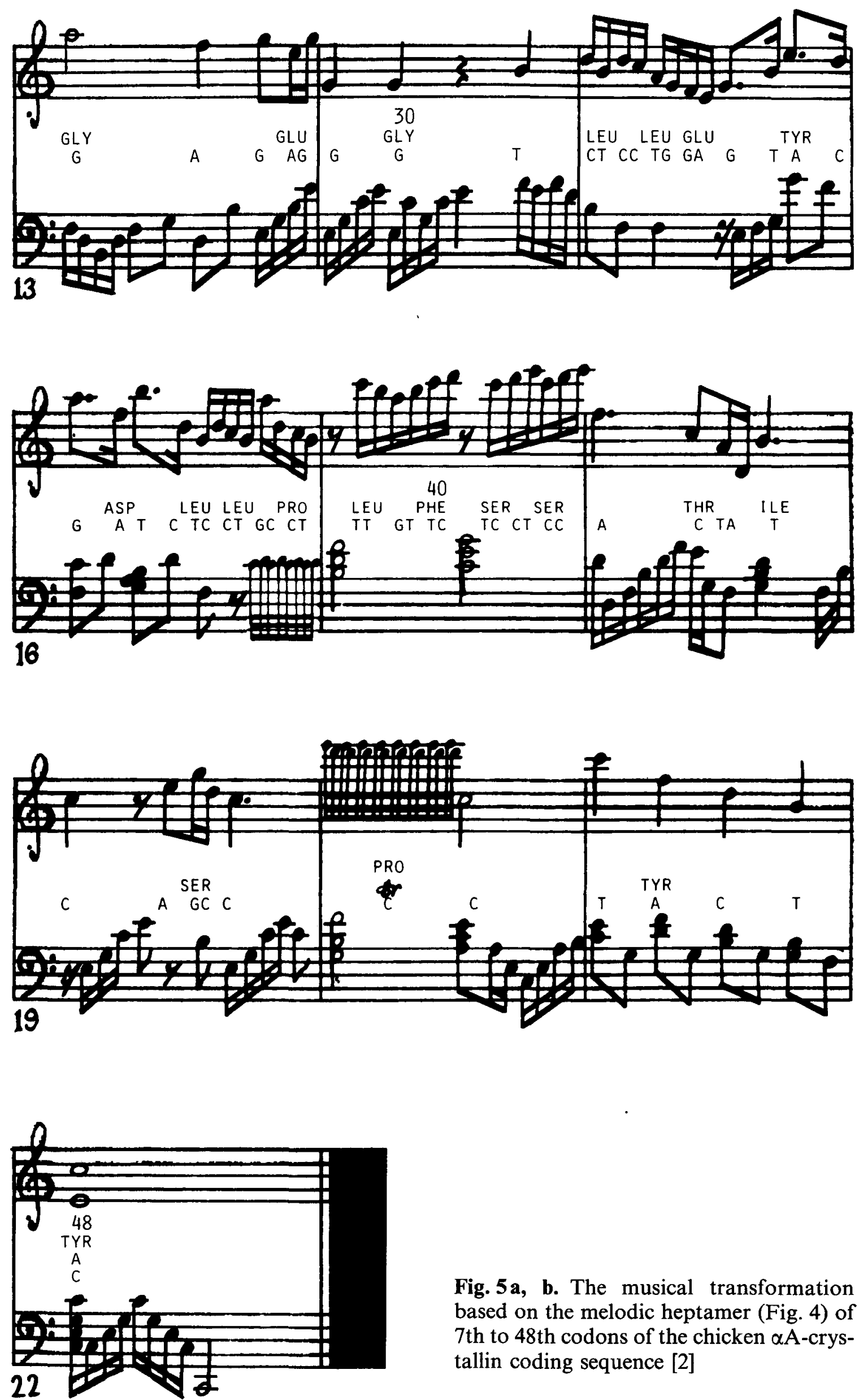

Fig. 5a, b. The musical transformation based on the melodic heptamer (Fig. 4) of 7 th to 48 th codons of the chicken $\alpha \mathrm{A}$-crystallin coding sequence [2] 
longer less exact periodicities. Indeed, two pairs of C C T G shown near the top of Fig. 2 were now separated by 3 and 5 bases.

\section{Periodicity Decay by the Golden Mean: the 3, 4, 7, 11, 18 Rule}

Of the consecutive numbers the first four are $1,2,3,4$. At this point, we begin to add previous two numbers to obtain the next number; i.e., $3+4=7$. If we keep doing this, the series of numbers form: 3 , $4,7,11,18,29,47,76,123$, etc. Now we divide 7 by 4,11 by 7,18 by 11 , and so on. Then we see that results begin to approach 1.618 and reach that goal at 123 divided by 76 , and remains 1.618 forever thereafter. Now, 1.618 is the well-known golden ratio expressed as

$\frac{a+b}{a}=\frac{a}{b}$ which is $\frac{1+\sqrt{5}}{2}$.

In a previous paper, we have shown that the periodicity decay in coding sequences is according to the above-noted golden mean [4]. Of the nine $\mathrm{C} \mathrm{C} \mathrm{T} \mathrm{G}$ tetramers, two recurred in immediate succession of each other as shown in Fig. 2. The remaining seven, on the other hand, recurred as parts of recurring heptamers. Two such pairs are shown in Fig. 3, because members of each pair are translated in different reading frames. Shown at the top row of Fig. 3 are two identical copies of the heptamer T Z C T C C T G, yielding the 80th and 81st SerPro when translated in the first reading frame, while encoding Leu-Leu dipeptide in the second reading frame. A pair of single-base-substituted copy T C T C G $\mathrm{G} \mathrm{G}$; the translation of this heptamer in its third reading frame encoding the 141 st to 143 rd Phe-Ser-Gly. It is pointed out that each of these five heptamers (one identical pair and a triplet derived from that pair) contained the second most frequently recurring base tetramer $\mathrm{T} \mathrm{C} \mathrm{T} \mathrm{C}$ already noted. Thus, five of the $7 \mathrm{X}$ recurring $\mathrm{T} \mathrm{C} \mathrm{T} \mathrm{Combined} \mathrm{with} \mathrm{the} \mathrm{most}$ frequently recurring tetramer $\mathrm{C} \mathrm{C} \mathrm{T} \mathrm{G}$ and its derivatives to become parts of recurring heptamers. Shown in the third row of Fig. 3 are another identical pair of C C T G-containing heptamers A C C C $T \mathrm{G}$ encoding the 16th and 17th Pro-Leu in its second reading frame, while encoding the seventh to tenth His-Pro-Trp in its third reading frame. This identical pair of heptamers on the one hand yielded its single-base-substituted derivatives (Fig. 3, fourth row) while, on the other hand, becoming parts of the pair of 11base-long repeating units that differed from each other by a single-base substitution (Fig. 3, bottom row). Thus, the periodicity decay by the chicken lens $\alpha$ Acrystallin coding sequence is indeed according to the golden mean: $4,7,11$ rule. Needless to say, single-base-substituted derivatives of the primordial heptamer $\mathrm{C}$ C T G have often become parts of the identical pair of heptamers. One such $G$ C T G-containing pair of identical heptamers encoding a pair of Met-Leu dipeptides of the 74th, 75th and 138th and 139th positions is shown in the fifth and sixth rows of Fig. 3.

When modern coding sequences are analyzed in the above manner, one can not help but realize that natural selection operating upon individual codons has mainly contributed to the conservation of a fait accompli by eliminating function-depriving, therefore, deleterious mutations. But this had very little to do with the initial acquisition of functions by proteins encoded by ancestral coding sequences of eons ago. For this, I contend that the universal principle of periodic-to-chaotic transition is responsible.

\section{Musical Transformation of the 7th to 48th Codons of the Chicken $\alpha$ A-Crystallin Coding Sequence}

Some time ago, I came to the realization that the periodic-to-chaotic transitional state of modern coding sequences can best be appreciated by their musical transformation under the set rule [5]. The 5 region of the 519-base-long chicken 
$\alpha$ A-crystallin coding sequence [2] is the domain ruled by the heptamers $\mathrm{A} \mathrm{C} \mathrm{C} \mathrm{C}$ C T G and T C T C C T G as shown in Fig. 3. By giving the melody shown in Fig. 4 to the former, the 7 th to 48 th codons of this coding sequence have been transformed to the musical composition for piano shown in Fig. 5a and b). By listening to it, one can readily realize the periodicity decay by the $4,7,11,18$ rule.

\section{E. Summary and Conclusions}

Modern coding sequences are in the periodic-to-chaotic transition. In the case of $\alpha \mathrm{A}$-crystallin coding sequence of the chicken, the initial tetrameric periodicity of the primordial tetramer C C T G has been decaying by the golden mean: the 4 , 7,11 rule. Thus, the tetramer has become parts of recurring heptamers, and some heptamers have become parts of the 11base-long repeating units.

\section{References}

1. Feigenbaum MJ (1985) The universal metric properties of nonlinear transformations. J Stat Physics 21:669-706

2. Okazaki KM, Yasuda $\mathrm{K}$, Kondoh $\mathrm{H}$, Okada TS (1985) DNA sequences responsible for tissue-specific expression of a chicken alpha-crystalling gene in mouse lens cells. EMBO J 4:2589-2595

3. Ohno S (1987) Evolution from primordial oligomeric repeats to modern coding sequences. J Mol Evol 25:325-329

4. Ohno S (1988) Codon preference is but an illusion created by the construction principle of coding sequences. Proc Natl Acad Sci USA $85 \mathrm{ff}$.

5. Ohno S, Ohno M (1986) The all pervasive principle of repetitious recurrence governs not only coding sequence construction but also human endeavor in musical composition. Immunogenetics $24: 71-78$ 